\title{
Masking Faults While Providing Bounded-Time Phased Recovery*
}

\author{
Borzoo Bonakdarpour and Sandeep S. Kulkarni \\ Department of Computer Science and Engineering \\ Michigan State University \\ East Lansing, MI 48824, USA \\ Email: $\{$ borzoo, sandeep\}@cse.msu.edu
}

\begin{abstract}
We focus on synthesis techniques for transforming existing fault-intolerant real-time programs to fault-tolerant programs that provide phased recovery. A fault-tolerant program is one that satisfies its safety and liveness specifications as well as timing constraints in the presence of faults. We argue that in many commonly considered programs (especially in mission-critical systems), when faults occur, simple recovery to the program's normal behavior is necessary, but not sufficient. For such programs, it is necessary that recovery is accomplished in a sequence of phases, each ensuring that the program satisfies certain properties. In this paper, we show that, in general, synthesizing fault-tolerant real-time programs that provide bounded-time phased recovery is NP-complete. We also characterize a sufficient condition for cases where synthesizing fault-tolerant real-time programs that provide bounded-time phased recovery can be accomplished in polynomial-time in the size of the input program's region graph.
\end{abstract}

\section{Keywords: Fault-tolerance, Real-time, Bounded-time recovery,} Phased recovery, Synthesis, Transformation, Formal methods.

\section{Motivation}

In this paper, we focus on the problem of automated synthesis for real-time systems that provide bounded-time phased recovery in the presence of faults. To illustrate this problem, first, we provide a motivating example to informally describe the idea of bounded-time phased recovery and the concepts of synthesis and fault-tolerance. We also use this example as a running demonstration throughout the paper.

Consider a one-lane turn-based bridge where cars can travel in only one direction at any time. The bridge is controlled by two traffic signals, say $s g_{0}$ and $s i g_{1}$, at the two ends of the bridge. The signals work as follows. Each signal

\footnotetext{
* This work was partially sponsored by NSF CAREER CCR-0092724 and ONR Grant N00014-01-1-0744.
} 
changes phase from green to yellow and then to red, based on a set of timing constraints. Moreover, if one signal is red, it will turn green some time after the other signal turns red. Thus, at any time, the values of $s i g_{0}$ and $s i g_{1}$ show in which direction cars are traveling. The specification of this system can be easily characterized by a set $S P E C_{b t}$ of bad transitions that reach states where both signals are not red at the same time. In order to address the correctness of the system, we identify a system invariant. Intuitively, the system invariant is a set $S$ of states from where the system behaves correctly. For example, in case of the traffic signals system, one system invariant is the set of states from where the system always reaches states where at least one signal is red and they change phases in time. Obviously, as long the system's state is in $S$, nothing catastrophic will happen. However, this is not the case when a system is subject to a set of faults.

Let us consider a scenario where the state of the systems is perturbed by occurrence of a fault that causes the system to reach a state, say $s$, in $\neg S$. Although reaching $s$ may not necessarily violate the system specification, subsequent signal operations can potentially result in execution of a transition in $S P E C_{b t}$. For example, when $s i g_{0}$ is green and $s i g_{1}$ is red, if the timer that is responsible for changing $\operatorname{sig}_{1}$ from red to green is reset due to a circuit problem, $s i g_{1}$ may turn green within some time while $s i g_{0}$ is also green. Such a system is called fault-intolerant, as it violates its specification in the presence of faults.

In order to transform this system into a fault-tolerant one, it is desirable to synthesize a version of the original system, in which even in the presence of faults, the system (1) never executes a transition $S P E C_{b t}$, and (2) always meets the following bounded-time recovery specification denoted by $S P E C_{b r}$ : When the system state is in $\neg S$, the system must reach a state in $S$ within a bounded amount of time. Although such a recovery mechanism is necessary in a fault-tolerant real-time system, it may not be sufficient. In particular, one may require that the system must initially reach a special set of states, say $Q$, within some time $\theta$, and subsequently recover to $S$ within $\delta$ time units. We call the set $Q$ an intermediate recovery predicate. The intuition for such phased recovery comes from the requirement that the occurrence of faults must be noted (e.g., for scheduling hardware repairs or replacement) before normal system operation resumes. Thus, in our example, $Q$ could be the set of states where all signals are red. Such a constraint ensures that the system first goes to a state in which a set of preconditions for final recovery (e.g., via a system reboot or rollback) is fulfilled.

In this paper, we concentrate on the problem of synthesizing real-time systems that provide bounded-time phased recovery in the presence of faults. Intuitively, the problem is as follows. After the occurrence of faults, the system must recover to a state in the set $Q$ within $\theta$ and from there, recover to the invariant $S$ within $\delta$ time units. The main results in this paper are as follows:

- We formally define the notion of bounded-time phased recovery in the context of fault-tolerant real-time systems. 
- We show that, in general (i.e., when $Q \nsubseteq S$ and $S \nsubseteq Q$ ), the problem of synthesizing fault-tolerant real-time programs that provide phased recovery is NP-complete. An example of such a case is the traffic signals system in which $Q$ includes states where all signals are flashing red.

- We characterize a sufficient condition for cases where the synthesis problem can be solved efficiently. In particular, we show that if $S \subseteq Q$, and, execution of the synthesized system needs to be closed in $Q$ (i.e., starting from a state in $Q$, the state of the system never leaves $Q$ ) then there exists a polynomialtime sound and complete synthesis algorithm in the size of time-abstract bisimulation of the input intolerant program. An example of such a case is the traffic signals system in which $Q$ is the set of states where either both signals remain red indefinitely or $S$ holds.

Organization of the paper. In Section 2, we formally define real-time programs and the type specifications that we consider in this paper. In Section 3, we present our fault model and introduce the notions of bounded-time phased recovery and fault-tolerance. We formally state the problem of synthesis of faulttolerant real-time programs that provide bounded-time phased recovery in Section 4. Then, in Section 5, we present our results on the complexity of the synthesis problem and the sufficient condition for existence of a polynomial-time sound and complete synthesis algorithm. In Section 6, we present the related work. Finally, in Section 7, we make concluding remarks and discuss future work.

\section{Real-Time Programs and Specifications}

In our framework, real-time programs are specified in terms of their state space and their transitions [AH97,AD94]. The definition of specification is adapted from Alpern and Schneider [AS85] and Henzinger [Hen92].

\subsection{Real-Time Program}

Let $V=\left\{v_{1}, v_{2} \cdots v_{n}\right\}, n \geq 1$, be a finite set of discrete variables and $X=$ $\left\{x_{1}, x_{2} \cdots x_{m}\right\}, m \geq 0$, be a finite set of clock variables. Each discrete variable $v_{i}, 1 \leq i \leq n$, is associated with a finite domain $D_{i}$ of values. Each clock variable $x_{j}, 1 \leq j \leq m$, ranges over nonnegative real numbers (denoted $\mathbb{R}_{\geq 0}$ ). A location is a function that maps discrete variables to a value from their respective domain. A clock constraint over the set $X$ of clock variables is a Boolean combination of formulae of the form $x \preceq c$ or $x-y \preceq c$, where $x, y \in X, c \in \mathbb{Z}_{\geq 0}$, and $\preceq$ is either $<$ or $\leq$. We denote the set of all clock constraints over $X$ by $\Phi(X)$. A clock valuation is a function $\nu: X \rightarrow \mathbb{R}_{\geq 0}$ that assigns a real value to each clock variable.

For $\tau \in \mathbb{R}_{\geq 0}$, we write $\nu+\tau$ to denote $\nu(x)+\tau$ for every clock variable $x$ in $X$. Also, for $\lambda \subseteq X, \nu[\lambda:=0]$ denotes the clock valuation that assigns 0 to each $x \in \lambda$ and agrees with $\nu$ over the rest of the clock variables in $X$. A state (denoted $\sigma$ ) is a pair $(s, \nu)$, where $s$ is a location and $\nu$ is a clock valuation for 
$X$. Let $u$ be a (discrete or clock) variable and $\sigma$ be a state. We denote the value of $u$ in state $\sigma$ by $u(\sigma)$. A transition is an ordered pair $\left(\sigma_{0}, \sigma_{1}\right)$, where $\sigma_{0}$ and $\sigma_{1}$ are two states. Transitions are classified into two types:

- Immediate transitions: $\left(s_{0}, \nu\right) \rightarrow\left(s_{1}, \nu[\lambda:=0]\right)$, where $s_{0}$ and $s_{1}$ are two locations, $\nu$ is a clock valuation, and $\lambda$ is a set of clock variables.

- Delay transitions: $(s, \nu) \rightarrow(s, \nu+\delta)$, where $s$ is a location, $\nu$ is a clock valuation, and $\delta \in \mathbb{R}_{\geq 0}$ is a time duration. We denote a delay transition of duration $\delta$ at state $\sigma$ by $(\sigma, \delta)$.

Thus, if $\psi$ is a set of transitions, we let $\psi^{s}$ and $\psi^{d}$ denote the set of immediate and delay transitions in $\psi$, respectively.

Definition 1 (real-time program) A real-time program $\mathcal{P}$ is a tuple $\left\langle S_{\mathcal{P}}, \psi_{\mathcal{P}}\right\rangle$, where $S_{\mathcal{P}}$ is the state space (i.e., the set of all possible states), and $\psi_{\mathcal{P}}$ is a set of transitions.

Definition 2 (state predicate) A state predicate $S$ is any subset of $S_{\mathcal{P}}$ such that in the corresponding Boolean expression, clock constraints are in $\Phi(X)$, i.e., clock variables are only compared with nonnegative integers.

By closure of a state predicate $S$ in a set $\psi_{\mathcal{P}}$ of transitions, we mean that (1) if an immediate transition originates in $S$ then it must terminate in $S$, and (2) if a delay transition originates in $S$ then it must remain in $S$ continuously.

Definition 3 (closure) A state predicate $S$ is closed in program $\mathcal{P}=\left\langle S_{\mathcal{P}}, \psi_{\mathcal{P}}\right\rangle$ (or briefly $\psi_{\mathcal{P}}$ ) iff

$$
\begin{aligned}
& \left(\forall\left(\sigma_{0}, \sigma_{1}\right) \in \psi_{\mathcal{P}}^{s}:\left(\left(\sigma_{0} \in S\right) \Rightarrow\left(\sigma_{1} \in S\right)\right)\right) \wedge \\
& \left(\forall(\sigma, \delta) \in \psi_{\mathcal{P}}^{d}:\left((\sigma \in S) \Rightarrow \forall \epsilon \mid\left(\left(\epsilon \in \mathbb{R}_{\geq 0}\right) \wedge(\epsilon \leq \delta)\right): \sigma+\epsilon \in S\right)\right) .
\end{aligned}
$$

Definition 4 (computation) A computation of $\mathcal{P}=\left\langle S_{\mathcal{P}}, \psi_{\mathcal{P}}\right\rangle$ (or briefly $\psi_{\mathcal{P}}$ ) is a finite or infinite timed state sequence of the form:

$$
\bar{\sigma}=\left(\sigma_{0}, \tau_{0}\right) \rightarrow\left(\sigma_{1}, \tau_{1}\right) \rightarrow \cdots
$$

iff the following conditions are satisfied: (1) $\forall j \in \mathbb{Z}_{\geq 0}:\left(\sigma_{j}, \sigma_{j+1}\right) \in \psi_{\mathcal{P}}$, (2) if $\bar{\sigma}$ is finite and terminates in $\left(\sigma_{f}, \tau_{f}\right)$ then there does not exist any state $\sigma$ such that $\left(\sigma_{f}, \sigma\right) \in \psi_{\mathcal{P}}^{s}$, and (3) the sequence $\tau_{0}, \tau_{1}, \cdots$ (called the global time), where $\tau_{i} \in \mathbb{R}_{\geq 0}$ for all $i \in \mathbb{Z}_{\geq 0}$, satisfies the following constraints:

1. (monotonicity) for all $i \in \mathbb{Z}_{\geq 0}, \tau_{i} \leq \tau_{i+1}$,

2. (divergence) if $\bar{\sigma}$ is infinite, for all $t \in \mathbb{R}_{\geq 0}$, there exists $j \in \mathbb{Z}_{\geq 0}$ such that $\tau_{j} \geq t$, and

3. (time consistency) for all $i \in \mathbb{Z}_{\geq 0}$, (1) if $\left(\sigma_{i}, \sigma_{i+1}\right)$ is a delay transition $\left(\sigma_{i}, \delta\right)$ in $\psi_{\mathcal{P}}^{d}$ then $\tau_{i+1}-\tau_{i}=\delta$, and $(2)$ if $\left(\sigma_{i}, \sigma_{i+1}\right)$ is an immediate transition in $\psi_{\mathcal{P}}^{s}$ then $\tau_{i}=\tau_{i+1}$.

We distinguish between a terminating finite computation and a deadlocked finite computation. Precisely, when a computation $\bar{\sigma}$ terminates in state $\sigma_{f}$, we include the delay transitions $\left(\sigma_{f}, \delta\right)$ in $\psi_{\mathcal{P}}^{d}$ for all $\delta \in \mathbb{R}_{\geq 0}$, i.e., $\bar{\sigma}$ can be extended to an infinite computation by advancing time arbitrarily. On the other hand, if there exists a state $\sigma_{d}$, such that there is no outgoing (delay or immediate) transition from $\sigma_{d}$ then $\sigma_{d}$ is a deadlock state. 


\subsection{Example}

As mentioned in Section 1, we use the one-lane bridge traffic controller as a running example throughout the paper. To concisely write the transitions of a program, we use timed guarded commands. A timed guarded command (also called timed action) is of the form $L:: g \stackrel{\lambda}{\rightarrow} s t$, where $L$ is a label, $g$ is a state predicate, st is a statement that describes how the program state is updated, and $\lambda$ is a set of clock variables that are reset by execution of $L$. Thus, $L$ denotes the set of transitions $\left\{\left(s_{0}, \nu\right) \rightarrow\left(s_{1}, \nu[\lambda:=0]\right) \mid g\right.$ is true in state $\left(s_{0}, \nu\right)$, and $s_{1}$ is obtained by changing $s_{0}$ as prescribed by $\left.s t\right\}$. A guarded wait command (also called delay action) is of the form $L:: g \longrightarrow$ wait, where $g$ identifies the set of states from where delay transitions with arbitrary durations are allowed to be taken as long as $g$ continuously remains true.

The one-lane bridge traffic controller program $(T C)$ has two discrete variables $\operatorname{sig}_{0}$ and $\operatorname{sig}_{1}$ with domain $\{G, Y, R\}$ to represent the status of signals. Moreover, for each signal $i, i \in\{0,1\}, T C$ has three clock variables $x_{i}, y_{i}$, and $z_{i}$ acting as timers to change signal phase. When a signal turns green, it may turn yellow within 10 time units, but not sooner than 1 time unit. Subsequently, the signal may turn red between 1 and 2 time units after it turns yellow. Finally, when the signal is red, it may turn green within 1 time unit after the other signal becomes red. Both signals operate identically. Thus, the traffic controller program is as follows. For $i \in\{0,1\}$ :

$$
\begin{array}{llll}
T C 1_{i}:: & \left(\operatorname{sig}_{i}=G\right) \wedge\left(1 \leq x_{i} \leq 10\right) & \stackrel{\left\{y_{i}\right\}}{\left.\longrightarrow z_{i}\right\}} & \left(\operatorname{sig}_{i}:=Y\right) ; \\
T C 2_{i}:: & \left(\operatorname{sig}_{i}=Y\right) \wedge\left(1 \leq y_{i} \leq 2\right) & \stackrel{\underline{\left\{z_{i}\right\}}}{\longrightarrow} & \left.\left(\operatorname{sig}_{i}:=R\right) ;=G\right) ; \\
T C 3_{i}:: & \left(\operatorname{sig}_{i}=R\right) \wedge\left(z_{j} \leq 1\right) & & \\
T C 4_{i}:: & \left(\left(\operatorname{sig}_{i}=G\right) \wedge\left(x_{i} \leq 10\right)\right) \vee & & \\
& \left(\left(\operatorname{sig}_{i}=Y\right) \wedge\left(y_{i} \leq 2\right)\right) \vee & & \\
& \left(\left(\operatorname{sig}_{i}=R\right) \wedge\left(z_{j} \leq 1\right)\right) & \longrightarrow & \text { wait; }
\end{array}
$$

where $j=(i+1) \bmod 2$. Notice that the guard of $T C 3_{i}$ depends on $z$ timer of signal $j$. For simplicity, we assume that once a traffic light turns green, all cars from the opposite direction have already left the bridge.

\subsection{Specification}

Let $\mathcal{P}=\left\langle S_{\mathcal{P}}, \psi_{\mathcal{P}}\right\rangle$ be a program. A specification (or property), denoted SPEC, for $\mathcal{P}$ is a set of infinite computations of the form $\left(\sigma_{0}, \tau_{0}\right) \rightarrow\left(\sigma_{1}, \tau_{1}\right) \rightarrow \cdots$ where $\sigma_{i} \in S_{\mathcal{P}}$ for all $i \in \mathbb{Z}_{\geq 0}$. Following Henzinger [Hen92], we require that all computations in SPEC satisfy time-monotonicity and divergence. We now define what it means for a program to satisfy a specification.

Definition 5 (satisfies) Let $\mathcal{P}=\left\langle S_{\mathcal{P}}, \psi_{\mathcal{P}}\right\rangle$ be a program, $S$ be a state predicate, and $S P E C$ be a specification for $\mathcal{P}$. We write $\mathcal{P} \models_{S} S P E C$ and say that $\mathcal{P}$ satisfies $S P E C$ from $S$ iff (1) $S$ is closed in $\psi_{\mathcal{P}}$, and (2) every computation of $\mathcal{P}$ that starts from $S$ is in $S P E C$. 
Definition 6 (invariant) Let $\mathcal{P}=\left\langle S_{\mathcal{P}}, \psi_{\mathcal{P}}\right\rangle$ be a program, $S$ be a state predicate, and $S P E C$ be a specification for $\mathcal{P}$. If $\mathcal{P} \models_{S} S P E C$ and $S \neq\{\}$, we say that $S$ is an invariant of $\mathcal{P}$ for $S P E C$.

Whenever the specification is clear from the context, we will omit it; thus, " $S$ is an invariant of $\mathcal{P}$ " abbreviates " $S$ is an invariant of $\mathcal{P}$ for $S P E C$ ". Note that Definition 5 introduces the notion of satisfaction with respect to infinite computations. In case of finite computations, we characterize them by determining whether they can be extended to an infinite computation in the specification.

Definition 7 (maintains) We say that program $\mathcal{P}$ maintains $S P E C$ from $S$ iff (1) $S$ is closed in $\psi_{\mathcal{P}}$, and (2) for all computation prefixes $\bar{\alpha}$ of $\mathcal{P}$, there exists a computation suffix $\bar{\beta}$ such that $\bar{\alpha} \bar{\beta} \in S P E C$. We say that $\mathcal{P}$ violates $S P E C$ iff it is not the case that $\mathcal{P}$ maintains $S P E C$.

Specifying timing constraints. In order to express time-related behaviors of real-time programs (e.g., deadlines and recovery time), we focus on a standard property typically used in real-time computing known as the bounded response property. A bounded response property, denoted $P \mapsto \leq \delta Q$ where $P$ and $Q$ are two state predicates and $\delta \in \mathbb{Z}_{\geq 0}$, is the set of all computations $\left(\sigma_{0}, \tau_{0}\right) \rightarrow$ $\left(\sigma_{1}, \tau_{1}\right) \rightarrow \cdots$ in which, for all $i \geq 0$, if $\sigma_{i} \in P$ then there exists $j, j \geq i$, such that (1) $\sigma_{j} \in Q$, and (2) $\tau_{j}-\tau_{i} \leq \delta$, i.e., it is always the case that a state in $P$ is followed by a state in $Q$ within $\delta$ time units.

The specifications considered in this paper are an intersection of a safety specification and a liveness specification [AS85,Hen92]. In this paper, we consider a special case where safety specification is characterized by a set of bad immediate transitions and a set of bounded response properties.

Definition 8 (safety specification) Let $S P E C$ be a specification. The safety specification of $S P E C$ is the union of the sets $S P E C_{\overline{b t}}$ and $S P E C_{\overline{b r}}$ defined as follows:

1. Let $S P E C_{b t}$ be a set of immediate bad transitions. We denote the specification whose computations have no transition in $S P E C_{b t}$ by $S P E C_{\overline{b t}}$.

2. We denote $S P E C_{b r}$ by the conjunction $\bigwedge_{i=0}^{m}\left(P_{i} \mapsto \leq \delta_{i} Q_{i}\right)$, for state predicates $P_{i}$ and $Q_{i}$, and, response times $\delta_{i}$.

Throughout the paper, $S P E C_{\overline{b r}}$ is meant to prescribe how a program should carry out bounded-time phased recovery to its normal behavior after the occurrence of faults. We formally define the notion of recovery in Section 3.

Definition 9 (liveness specification) A liveness specification of $S P E C$ is a set of computations that meets the following condition: for each finite computation $\bar{\alpha} \in S P E C$, there exists a computation $\bar{\beta}$ such that $\bar{\alpha} \bar{\beta} \in S P E C$.

Remark 1. In our synthesis problem in Section 4, we begin with an initial program that satisfies its specification (including the liveness specification). We will show that our synthesis techniques preserve the liveness specification. Hence, the liveness specification need not be specified explicitly. 


\subsection{Example (cont'd)}

Following Definition 8, the safety specification of $T C$ comprises of $S P E C_{b t_{T C}}$ and $S P E C_{b r_{T C}} . S P E C_{b t_{T C}}$ is simply the set of transitions where both signals are not red in their target states:

$$
\operatorname{SPEC}_{b t_{T C}}=\left\{\left(\sigma_{0}, \sigma_{1}\right) \mid\left(\operatorname{sig}_{0}\left(\sigma_{1}\right) \neq R\right) \wedge\left(\operatorname{sig}_{1}\left(\sigma_{1}\right) \neq R\right)\right\} .
$$

We define $S P E C_{b r}$ of $T C$ in Section 3, where we formally define the notion of bounded-time phased recovery.

One invariant for the program $T C$ is the following:

$$
\begin{aligned}
S_{T C}=\forall i \in\{0,1\}: & {\left[\left(\operatorname{sig}_{i}=G\right) \Rightarrow\left(\left(\operatorname{sig}_{j}=R\right) \wedge\left(x_{i} \leq 10\right) \wedge\left(z_{i}>1\right)\right)\right] \wedge } \\
& {\left[\left(\operatorname{sig}_{i}=Y\right) \Rightarrow\left(\left(\operatorname{sig}_{j}=R\right) \wedge\left(y_{i} \leq 2\right) \wedge\left(z_{i}>1\right)\right)\right] \wedge } \\
& {\left[\left(\operatorname{sig}_{i}=R\right) \wedge\left(\operatorname{sig}_{j}=R\right)\right) } \\
& \left.\Rightarrow\left(\left(z_{i} \leq 1\right) \oplus\left(z_{j} \leq 1\right)\right)\right],
\end{aligned}
$$

where $j=(i+1) \bmod 2$ and $\oplus$ denotes the exclusive or operator. It is straightforward to see that $T C$ satisfies $S P E C_{\overline{b t}_{T C}}$ from $S_{T C}$.

\section{Fault Model and Fault-Tolerance}

\subsection{Fault Model}

The faults that a program is subject to are systematically represented by transitions. A class of faults $f$ for program $\mathcal{P}=\left\langle S_{\mathcal{P}}, \psi_{\mathcal{P}}\right\rangle$ is a subset of immediate and delay transitions of the set $S_{\mathcal{P}} \times S_{\mathcal{P}}$. We use $\psi_{\mathcal{P}}[] f$ to denote the transitions obtained by taking the union of the transitions in $\psi_{\mathcal{P}}$ and the transitions in $f$.

Definition 10 (fault-span) We say that a state predicate $T$ is an $f$-span (read as fault-span) of $\mathcal{P}=\left\langle S_{\mathcal{P}}, \psi_{\mathcal{P}}\right\rangle$ from $S$ iff the following conditions are satisfied: (1) $S \subseteq T$, and (2) $T$ is closed in $\psi_{\mathcal{P}}[] f$.

Example (cont'd). $\quad T C$ is subject to clock reset faults due to circuit malfunctions. In particular, we consider faults that reset either $z_{0}$ or $z_{1}$ at any state in the invariant $S_{T C}$ (cf. Subsection 2.3), without changing the location of $T C$ :

$$
\begin{array}{lllll}
F_{0}:: & S_{T C} & \stackrel{\left\{z_{0}\right\}}{\longrightarrow} & \text { skip; } \\
F_{1}:: & S_{T C} & \stackrel{\left\{z_{1}\right\}}{\longrightarrow} & \text { skip; }
\end{array}
$$

It is straightforward to see that in the presence of $F_{0}$ and $F_{1}, T C$ may violate $S P E C_{\overline{b t}_{T C}}$. For instance, if $F_{1}$ occurs when $T C$ is in a state of $S_{T C}$ where $\left(\operatorname{sig}_{0}=\operatorname{sig}_{1}=R\right) \wedge\left(z_{0} \leq 1\right) \wedge\left(z_{1}>1\right)$, in the resulting state, we have $\left(\operatorname{sig}_{0}=\right.$ $\left.\operatorname{sig}_{1}=R\right) \wedge\left(z_{0} \leq 1\right) \wedge\left(z_{1}=0\right)$. From this state, immediate execution of timed actions $T C 3_{0}$ and then $T C 3_{1}$ results in a state where $\left(s i g_{0}=s i g_{1}=G\right)$, which is clearly a violation of the safety specification. 


\subsection{Phased Recovery and Fault-Tolerance}

As illustrated in Section 1, preserving safety specification and providing simple recovery to the invariant from the fault-span may not be sufficient and, hence, it may be necessary to complete recovery to the invariant in a sequence of phases where each phase satisfies certain constraints. We formalize the notion of bounded-time phased recovery by a set of bounded response properties inside the safety specification, i.e., by $S P E C_{b r}$ (cf. Definition 8). In this paper, in particular, we focus on 2-phase recovery.

Definition 11 (2-phase recovery) Let $\mathcal{P}=\left\langle S_{\mathcal{P}}, \psi_{\mathcal{P}}\right\rangle$ be a real-time program with invariant $S, Q$ be an arbitrary intermediate recovery predicate, $f$ be a set of faults, and $S P E C$ be a specification (as defined in Definitions 8 and 9). We say that $\mathcal{P}$ provides 2 -phase recovery from $S$ and $Q$ with recovery times $\delta, \theta \in$ $\mathbb{Z}_{\geq 0}$, respectively, iff $\left\langle S_{\mathcal{P}}, \psi_{\mathcal{P}}[] f\right\rangle$ maintains $S P E C_{\overline{b r}}$ from $S$, where $S P E C_{\overline{b r}} \equiv$ $(\neg S \mapsto \leq \theta Q) \wedge(Q \mapsto \leq \delta S)$.

Note that in Definition 11, if $S$ and $Q$ are disjoint then $\mathcal{P}$ has to recover to $Q$ and then $S$ in order, as $S$ is closed in $\mathcal{P}$. On the other hand, if $S$ and $Q$ are not disjoint, $\mathcal{P}$ has the following options: (1) recover to $Q \cap \neg S$ within $\theta$ and then $S$, or (2) directly recover to $S \cap Q$ within $\min (\delta, \theta)$.

We are now ready to define what it means for a program to be fault-tolerant while providing 2-phase recovery. Intuitively, a fault-tolerant program satisfies its safety, liveness, and timing constraints in both absence and presence of faults. In other words, the program masks the occurrence of faults in the sense that all program requirements are persistently met in both absence and presence of faults.

Definition 12 (fault-tolerance) Let $\mathcal{P}=\left\langle S_{\mathcal{P}}, \psi_{\mathcal{P}}\right\rangle$ be a real-time program with invariant $S, f$ be a set of faults, and $S P E C$ be a specification as defined in Definitions 8 and 9. We say that $\mathcal{P}$ is $f$-tolerant to $S P E C$ from $S$, iff (1) $\mathcal{P} \models_{S} S P E C$, and (2) there exists $T$ such that $T$ is an $f$-span of $\mathcal{P}$ from $S$ and $\left\langle S_{\mathcal{P}}, \psi_{\mathcal{P}}[] f\right\rangle$ maintains $S P E C$ from $T$.

Notation. Whenever the specification $S P E C$ and the invariant $S$ are clear from the context, we omit them; thus, " $f$-tolerant" abbreviates " $f$-tolerant to $S P E C$ from $S "$.

Example (cont'd). As described in Section 1, when faults $F_{0}$ or $F_{1}$ (defined in Subsection 3.1) occur, the program $T C$ has to, first, ensure that nothing catastrophic happens and then recover to its normal behavior. Thus, the faulttolerant version of $T C$ has to, first, reach a state where both signals remain red indefinitely and subsequently recover to $S$ where exactly one signal turns green. In particular, we let the 2-phase recovery specification of $T C$ be the following:

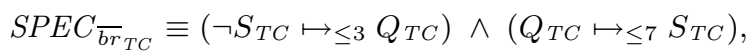

where $Q_{T C}=\forall i \in\{0,1\}:\left(\operatorname{sig}_{i}=R\right) \wedge\left(z_{i}>1\right)$. The response times in $S P E C_{b r_{T C}}$ (i.e., 3 and 7 ) are simply two arbitrary numbers to express the duration of the two phases of recovery. 


\section{Problem Statement}

Given are a fault-intolerant real-time program $\mathcal{P}=\left\langle S_{\mathcal{P}}, \psi_{\mathcal{P}}\right\rangle$, its invariant $S$, a set $f$ of faults, and a specification $S P E C$ such that $\mathcal{P} \models_{S} S P E C$. Our goal is to synthesize a real-time program $\mathcal{P}^{\prime}=\left\langle S_{\mathcal{P}^{\prime}}, \psi_{\mathcal{P}^{\prime}}\right\rangle$ with invariant $S^{\prime}$ such that $\mathcal{P}^{\prime}$ is $f$-tolerant to $S P E C$ from $S^{\prime}$. We require that our synthesis methods obtain $\mathcal{P}^{\prime}$ from $\mathcal{P}$ by adding fault-tolerance to $\mathcal{P}$ without introducing new behaviors in the absence of faults. To this end, we first define the notion of projection. Projection of a set $\psi_{\mathcal{P}}$ of transitions on state predicate $S$ consists of immediate transitions of $\psi_{\mathcal{P}}^{s}$ that start in $S$ and end in $S$, and delay transitions of $\psi_{\mathcal{P}}^{d}$ that start and remain in $S$ continuously.

Definition 13 (projection) Projection of a set $\psi$ of transitions on a state predicate $S$ (denoted $\psi \mid S$ ) is the following set of transitions:

$$
\begin{aligned}
\psi \mid S= & \left\{\left(\sigma_{0}, \sigma_{1}\right) \in \psi^{s} \mid \sigma_{0}, \sigma_{1} \in S\right\} \cup \\
& \left\{(\sigma, \delta) \in \psi^{d} \mid \sigma \in S \wedge\left(\forall \epsilon \mid\left(\left(\epsilon \in \mathbb{R}_{\geq 0}\right) \wedge(\epsilon \leq \delta)\right): \sigma+\epsilon \in S\right)\right\} .
\end{aligned}
$$

Since meeting timing constraints in the presence of faults requires time predictability, we let our synthesis methods incorporate a finite set $Y$ of new clock variables. We denote the set of states obtained by abstracting the clock variables in $Y$ from the state predicate $U$ by $U \backslash Y$. Likewise, if $\psi$ is a set of transitions, we denote the set of transitions obtained by abstracting the clock variables in $Y$ by $\psi_{\mathcal{P}} \backslash Y$. Now, observe that in the absence of faults, if $S^{\prime}$ contains states that are not in $S$ then $\mathcal{P}^{\prime}$ may include computations that start outside $S$. Hence, we require that $\left(S^{\prime} \backslash Y\right) \subseteq S$. Moreover, if $\psi_{\mathcal{P}}^{\prime} \mid S^{\prime}$ contains a transition that is not in $\psi_{\mathcal{P}} \mid S^{\prime}$ then in the absence of faults, $\mathcal{P}^{\prime}$ can exhibit computations that do not correspond to computations of $\mathcal{P}$. Therefore, we require that $\left(\psi_{\mathcal{P}^{\prime}} \backslash Y\right)\left|\left(S^{\prime} \backslash Y\right) \subseteq \psi_{\mathcal{P}}\right|\left(S^{\prime} \backslash Y\right)$.

Problem Statement 1 Given a program $\mathcal{P}=\left\langle S_{\mathcal{P}}, \psi_{\mathcal{P}}\right\rangle$, invariant $S$, specification $S P E C$, and set of faults $f$ such that $\mathcal{P} \models_{S} S P E C$, identify $\mathcal{P}^{\prime}=\left\langle S_{\mathcal{P}^{\prime}}, \psi_{\mathcal{P}^{\prime}}\right\rangle$ and $S^{\prime}$ such that:

(C1) $S_{\mathcal{P}^{\prime}} \backslash Y=S_{\mathcal{P}}$, where $Y$ is a finite set of new clock variables,

(C2) $\left(S^{\prime} \backslash Y\right) \subseteq S$,

(C3) $\quad\left(\left(\psi_{\mathcal{P}^{\prime}} \backslash Y\right) \mid\left(\left(S^{\prime} \backslash Y\right)\right) \subseteq\left(\psi_{\mathcal{P}} \mid\left(S^{\prime} \backslash Y\right)\right)\right.$, and

$(C 4) \quad \mathcal{P}^{\prime}$ is $f$-tolerant to $S P E C$ from $S^{\prime}$.

\section{Synthesizing Fault-Tolerant Real-Time Programs with 2-Phase Recovery}

\subsection{Complexity}

In this section, we show that, in general, the problem of synthesizing faulttolerant real-time programs that provide phased recovery is NP-complete in the size of locations of the given fault-intolerant real-time program. 
Instance. A real-time program $\mathcal{P}=\left\langle S_{\mathcal{P}}, \psi_{\mathcal{P}}\right\rangle$ with invariant $S$, a set of faults $f$, and a specification $S P E C$, such that $\mathcal{P} \models_{S} S P E C$, where $S P E C_{b r} \equiv\left(\neg S \mapsto_{\leq \theta}\right.$ $Q) \wedge(Q \mapsto \leq \delta S)$ for state predicate $Q$ and $\delta, \theta \in \mathbb{Z}_{\geq 0}$.

The decision problem (FTPR). Does there exist an $f$-tolerant program $\mathcal{P}^{\prime}=\left\langle S_{\mathcal{P}^{\prime}}, \psi_{\mathcal{P}^{\prime}}\right\rangle$ with invariant $S^{\prime}$ such that $\mathcal{P}^{\prime}$ and $S^{\prime}$ meet the constraints of Problem Statement 1?

Theorem 1. The FTPR problem is NP-complete in the size of locations of the fault-intolerant program.

Example (cont'd). The proof of Theorem 1 particularly implies that if $Q$ and $S$ are disjoint in the problem instance then NP-completeness of the synthesis problem is certain. In the context of $T C$, notice that according to the definitions of $S_{T C}$ and $Q_{T C}$ in Subsections 2.4 and 3.2, it is the case that $S_{T C} \cap Q_{T C}=$ \{\} . Hence, the $T C$ program and specification in their current form exhibit an instance where the synthesis problem is NP-complete. However, in Subsection 5.3 , we demonstrate that a slight modification in the specification of $T C$ makes the problem significantly easier to solve.

\subsection{A Sufficient Condition for a Polynomial-Time Solution}

In this section, we present a sufficient condition under which one can devise a polynomial-time sound and complete solution to the Problem Statement 1 in the size of time-abstract bisimulation of input program.

Claim. Let $\mathcal{P}=\left\langle S_{\mathcal{P}}, \psi_{\mathcal{P}}\right\rangle$ be a program with invariant $S$ and recovery specification $S P E C_{\overline{b r}} \equiv(\neg S \mapsto \leq \theta Q) \wedge(Q \mapsto \leq \delta S)$. There exists a polynomial-time sound and complete solution to Problem Statement 1 in the size of the region graph of $\mathcal{P}$, if $(S \subseteq Q) \wedge\left(Q\right.$ is closed in $\left.\psi_{\mathcal{P}^{\prime}}\right)$.

In order to validate this claim, we propose the Algorithm Add_BoundedPhasedRecovery.

Algorithm sketch. Intuitively, the algorithm works as follows. In Step 1, we transform the input program into a region graph [AD94] (described below). In Step 2, we isolate the set of states from where $S P E C_{\overline{b t}}$ may be violated. In Step 3, we ensure that any computation of $\mathcal{P}^{\prime}$ that starts from a state in $\neg S^{\prime}-Q$ (respectively, $Q-S^{\prime}$ ) reaches a state in $Q$ (respectively, $S^{\prime}$ ) within $\theta$ (respectively, $\delta$ ) time units. In Step 4, we ensure the closure of fault-span and deadlock freedom of invariant. We repeat Steps 3-4 until a fixpoint is reached. Finally, in Step 5, we transform the resultant region graph back into a real-time program.

Assumption 1 Let $\bar{\alpha}=\left(\sigma_{0}, \tau_{0}\right) \rightarrow\left(\sigma_{1}, \tau_{1}\right) \rightarrow \cdots\left(\sigma_{n}, \tau_{n}\right)$ be a computation prefix where $\sigma_{0}, \sigma_{n} \in S$ and $\sigma_{i} \notin S$ for all $i \in\{1 . . n-1\}$. Only for simplicity of presentation, we assume that the number of occurrence of faults in $\bar{\alpha}$ is one. Precisely, we assume that in $\bar{\alpha}$, only $\left(\sigma_{0}, \sigma_{1}\right)$ is a fault transition and no faults occur outside the program invariant. In our previous work [BK06b], we have shown 
how to deal with cases where multiple faults occur in a computation when adding bounded response properties. The same technique can be applied while preserving soundness and completeness of the algorithm Add_BoundedPhasedRecovery in this paper. Furthermore, notice that the proof of Theorem 1 in its current form holds with this assumption.

Region Graph. Real-time programs can be analyzed with the help of an equivalence relation of finite index on the set of states [AD94]. Given a realtime program $\mathcal{P}$, for each clock variable $x \in X$, let $c_{x}$ be the largest constant in clock constraint of transitions of $p$ that involve $x$, where $c_{x}=0$ if $x$ does not occur in any clock constraints of $\mathcal{P}$. We say that two clock valuations $\nu$, $\mu$ are clock equivalent if (1) for all $x \in X$, either $\lfloor\nu(x)\rfloor=\lfloor\mu(x)\rfloor$ or both $\nu(x), \mu(x)>c_{x},(2)$ the ordering of the fractional parts of the clock variables in the set $\left\{x \in X \mid \nu(x)<c_{x}\right\}$ is the same in $\mu$ and $\nu$, and (3) for all $x \in X$ where $\nu(x)<c_{x}$, the clock value $\nu(x)$ is an integer iff $\mu(x)$ is an integer. A clock region $\rho$ is a clock equivalence class. Two states $\left(s_{0}, \nu_{0}\right)$ and $\left(s_{1}, \nu_{1}\right)$ are region equivalent, written $\left(s_{0}, \nu_{0}\right) \equiv\left(s_{1}, \nu_{1}\right)$, if (1) $s_{0}=s_{1}$, and (2) $\nu_{0}$ and $\nu_{1}$ are clock equivalent. A region $r=(s, \rho)$ is an equivalence class with respect to $\equiv$, where $s$ is a location and $\rho$ is a clock region. We say that a clock region $\beta$ is a time-successor of a clock region $\alpha$ iff for each $\nu \in \alpha$, there exists $\tau \in \mathbb{R}_{\geq 0}$, such that $\nu+\tau \in \beta$, and $\nu+\tau^{\prime} \in \alpha \cup \beta$ for all $\tau^{\prime}<\tau$.

Using the region equivalence relation, we construct the region graph of $\mathcal{P}=$ $\left\langle S_{\mathcal{P}}, \psi_{\mathcal{P}}\right\rangle\left(\right.$ denoted $\left.R(\mathcal{P})=\left\langle S_{\mathcal{P}}^{r}, \psi_{\mathcal{P}}^{r}\right\rangle\right)$ as follows. Vertices of $R(\mathcal{P})$ (denoted $S_{\mathcal{P}}^{r}$ ) are regions. Edges of $R(\mathcal{P})$ (denoted $\psi_{\mathcal{P}}^{r}$ ) are of the form $\left(s_{0}, \rho_{0}\right) \rightarrow\left(s_{1}, \rho_{1}\right)$ iff for some clock valuations $\nu_{0} \in \rho_{0}$ and $\nu_{1} \in \rho_{1},\left(s_{0}, \nu_{0}\right) \rightarrow\left(s_{1}, \nu_{1}\right)$ is a transitions in $\psi_{\mathcal{P}}$.

We now describe the algorithm Add_BoundedPhasedRecovery in detail:

- (Step 1) First, we use the above technique to transform the input program $\mathcal{P}=\left\langle S_{\mathcal{P}}, \psi_{\mathcal{P}}\right\rangle$ into a region graph $R(\mathcal{P})=\left\langle S_{\mathcal{P}}^{r}, \psi_{\mathcal{P}}^{r}\right\rangle$. To this end, we invoke the procedure ConstructRegionGraph as a black box (Line 1). We let this procedure convert state predicates and sets of transitions in $\mathcal{P}$ (e.g., $S$ and $\psi_{\mathcal{P}}$ ) to their corresponding region predicates and sets of edges in $R(\mathcal{P})$ (e.g., $S^{r}$ and $\left.\psi_{\mathcal{P}}^{r}\right)$. Precisely, a region predicate $U^{r}$ with respect to a state predicate $U$ is the set $U^{r}=\{(s, \rho) \mid \exists(s, \nu):((s, \nu) \in U \wedge \nu \in \rho)\}$.

- (Step 2) In order to ensure that the synthesized program does not violate $S P E C_{\overline{b t}}$, we identify the set $m s$ of regions from where a computation may reach a transition in $S P E C_{b t}$ by taking fault transitions alone (Line 2). Next (Line 3), we compute the set $m t$ of edges, which contains (1) edges that directly violate safety (i.e., $S P E C_{b t}^{r}$ ), and (2) edges whose target region is in $m s$ (i.e., edges that lead a computation to a state from where safety may be violated by faults alone). Since the program does not have control over occurrence of faults, we remove the set $m s$ from the region predicate $T_{1}^{r}$, which is our initial estimate of the fault-span (Line 4). Likewise, in Step 3 , we will remove $m t$ from the set of program edges $\psi_{\mathcal{P}}^{r}$ when recomputing program transitions. 


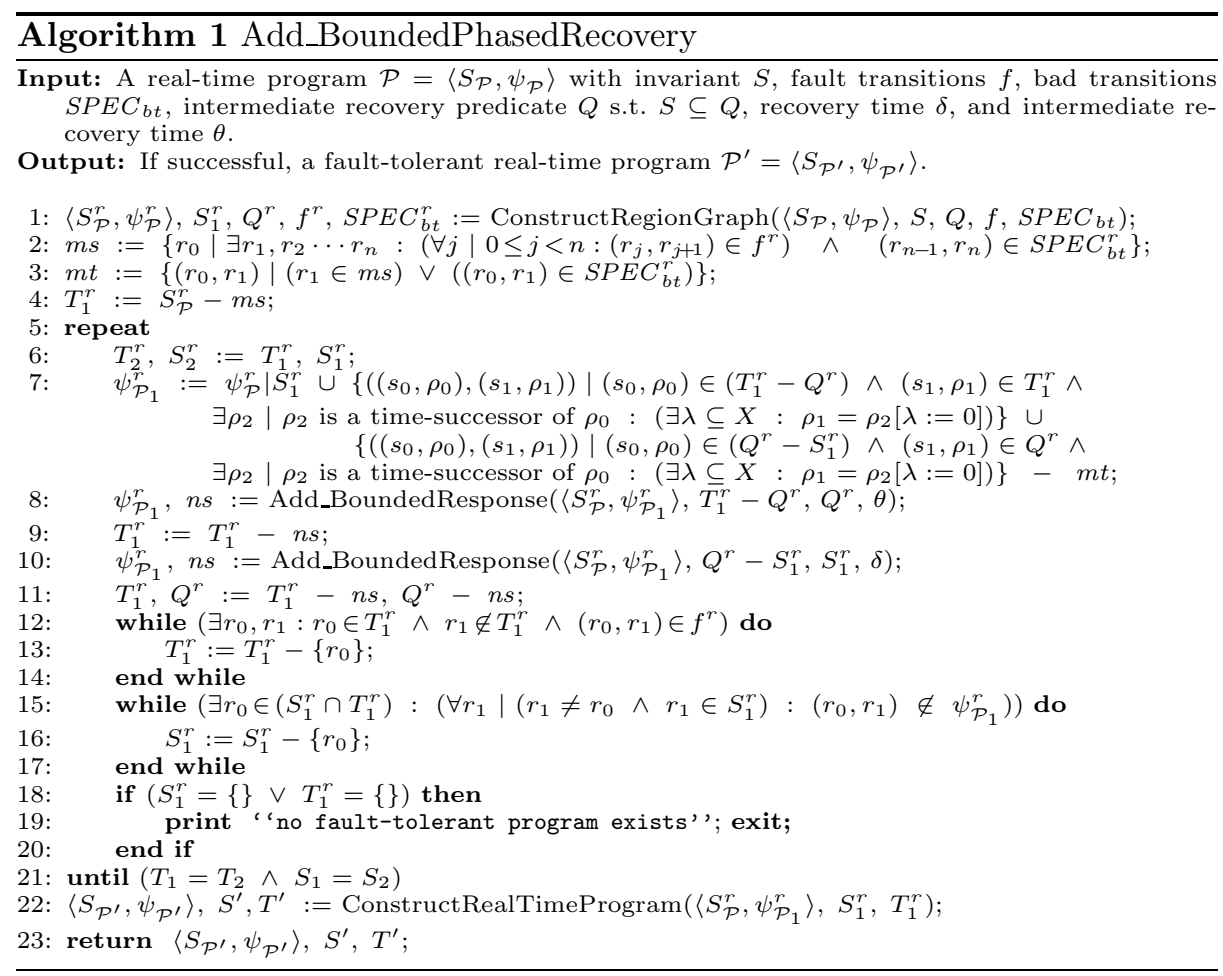

- (Step 3) In this step, we add recovery paths to $R(\mathcal{P})$ so that $R(\mathcal{P})$ satisfies

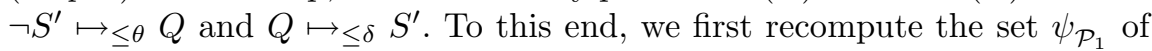
program edges (Line 7) by including (1) existing edges that start and end in $S_{1}^{r}$, and (2) new recovery edges that originate from regions in $T_{1}^{r}-Q^{r}$ (respectively, $Q^{r}-S_{1}^{r}$ ) and terminate at regions in $T_{1}^{r}$ (respectively, $Q$ ) such that the time-monotonicity condition is met. We exclude the set $m t$ from $\psi_{\mathcal{P}_{1}}^{r}$ to ensure that these recovery edges do not violate $S P E C_{\overline{b t}}$. Notice that the algorithm allows arbitrary clock resets during recovery. If such clock resets are not desirable, one can rule them out by including them as bad transitions in $S P E C_{b t}$.

After adding recovery edges, we invoke the procedure Add_BoundedResponse (Line 8) with parameters $T_{1}^{r}-Q^{r}, Q^{r}$, and $\theta$ to ensure that $R(\mathcal{P})$ indeed satisfies the bounded response property $\neg S \mapsto \leq \theta Q$. The details of how the procedure Add_BoundedResponse (first proposed in [BK06a]) functions are not provided in this paper, with the exception of the following properties: (1) it adds a clock variable, say $t_{1}$, which gets reset when $T_{1}-Q$ becomes true, to the set $X$ of clock variables of $\mathcal{P},(2)$ for each state $\sigma$ in $T_{1}-Q$, it includes the set of transitions that participate in forming the computation that starts from $\sigma$ and reaches a state in $Q$ with smallest possible time delay, if the delay is less than $\theta$, and (3) the regions made unreachable by this procedure (returned as the set $n s$ ) cannot be present in any solution 
that satisfies $\neg S_{1} \mapsto \leq \theta Q$. The procedure may optionally include additional computations, provided they preserve the corresponding bounded response property. Thus, since there does not exist a computation prefix that maintains the corresponding bounded response property from the regions in $n s$, in Line 9, the algorithm removes $n s$ from $T_{1}^{r}$. Likewise, in Line 10, the algorithm adds a clock variable, say $t_{2}$, which gets reset when $Q-S_{1}$ becomes true and ensures that $R(\mathcal{P})$ satisfies $Q \mapsto \leq \delta S_{1}$.

- (Step 4) Since we remove the set $n s$ of regions from $T_{1}^{r}$, we need to ensure that $T_{1}$ is closed in $f$. Thus, we remove regions from where a sequence of fault edges can reach a region in $n s$ (Lines 12-14). Next, due to the possibility of removal of some regions and edges in the previous steps, the algorithm ensures that the region graph $\left\langle S_{\mathcal{P}}^{r}, \psi_{\mathcal{P}_{1}}^{r}\right\rangle$ does not have deadlock regions in the region invariant $S_{1}^{r}$ (Lines 15-17). Precisely, we say that a region $\left(s_{0}, \rho_{0}\right)$ of region graph $R(\mathcal{P})=\left\langle S_{\mathcal{P}}^{r}, \psi_{\mathcal{P}}^{r}\right\rangle$ is a deadlock region in region predicate $U^{r}$ iff for all regions $\left(s_{1}, \rho_{1}\right) \in U^{r}$, there does not exist an edge of the form $\left(s_{0}, \rho_{0}\right) \rightarrow\left(s_{1}, \rho_{1}\right) \in \psi_{\mathcal{P}}^{r}$. Deadlock freedom in the region graph is necessary, as the constraint $C 4$ in the Problem Statement 1 does not allow the algorithm to introduce new finite or time-divergent computations to the input program. If the removal of deadlock regions and regions from where the closure of fault-span is violated results in empty invariant or fault-span, the algorithm declares failure (Lines 18-20).

- (Step 5) Finally, upon reaching a fixpoint, we transform the resulting region graph $\left\langle S_{\mathcal{P}}^{r}, \psi_{\mathcal{P}_{1}}^{r}\right\rangle$ back into a real-time program $\mathcal{P}^{\prime}=\left\langle S_{\mathcal{P}^{\prime}}, \psi_{\mathcal{P}^{\prime}}\right\rangle$ by invoking the procedure ConstructRealTimeProgram. In fact, the program $\mathcal{P}^{\prime}$ is returned as the final synthesized fault-tolerant program. Note that since a region graph is a time-abstract bisimulation [AD94], we will not lose any behaviors in the reverse transformation.

Theorem 2. The Algorithm Add_BoundedPhasedRecovery is sound and complete.

\subsection{Example (cont'd)}

We now demonstrate how the algorithm Add_BoundedPhasedRecovery synthesizes a fault-tolerant version of $T C$, which provides bounded-time recovery. Let the intermediate recovery predicate be:

$$
Q_{\text {new }}=S_{T C} \cup Q_{T C} .
$$

In other words, after the occurrence of faults, the recovery specification requires that either both signals turn red within 3 time units and then return to the normal behavior within 7 time units, or, the system reaches a state in $S_{T C}$ within 3 time units. Since, $S_{T C} \subseteq Q_{\text {new }}$, we apply the Algorithm Add_BoundedPhasedRecovery to transform $T C$ into a fault-tolerant program $T C^{\prime}$. We note that due to many symmetries in $T C$ and the complex structure of the algorithm, we only present a highlight of the process of synthesizing $T C^{\prime}$. 
First, observe that in Step 2 of the algorithm, $m s=\{\}$ and $m t=S P E C_{b t_{T C}}$. In Step 3, consider a subset of $T_{1}-Q_{\text {new }}$ where $\left(\operatorname{sig}_{0}=\operatorname{sig}_{1}=R\right) \wedge\left(z_{0}, z_{1} \leq 1\right)$. This predicate is reachable by a single occurrence of (for instance) $F_{0}$ from an invariant state where $\left(\operatorname{sig}_{0}=\operatorname{sig}_{1}=R\right) \wedge\left(z_{0}>1\right) \wedge\left(z_{1} \leq 1\right)$. After adding legitimate recovery transitions (Line 7 ), the invocation of Add_BoundedResponse (Line 8) results in addition of the following recovery action:

$$
T C 5_{i}:: \quad\left(\operatorname{sig}_{0}=\operatorname{sig}_{1}=R\right) \wedge\left(z_{0}, z_{1} \leq 2\right) \wedge\left(t_{1} \leq 2\right) \quad \longrightarrow \quad \text { wait; }
$$

for all $i \in\{0,1\}$. This action enforces the program to take delay transitions so that the program reaches a state in $Q$ where $\left(\operatorname{sig}_{0}=\operatorname{sig}_{1}=R\right) \wedge\left(z_{0}, z_{1}>1\right)$.

Now, consider the case where $T C$ is in a state where $\left(\operatorname{sig}_{0}=G\right) \wedge\left(\operatorname{sig}_{1}=\right.$ $R) \wedge\left(x_{0}=1\right) \wedge\left(z_{0}, z_{1} \leq 1\right)$. In this case, one may argue that $T C$ has the option of executing action $T C 3_{1}$ and reaching a state where $s i g_{0}=s i g_{1}=G$, which is clearly a violation of safety specification $S P E C_{\overline{b t}_{T C}}$. However, since we remove the set $m t$ from $\psi_{\mathcal{P}_{1}}$ (Line 7 ), action $T C 3_{i}$ would be revised as follows:

$$
T C 3_{i}:: \quad\left(\operatorname{sig}_{i}=R\right) \wedge\left(z_{j} \leq 1\right) \wedge\left(\operatorname{sig}_{j} \neq G\right) \quad \stackrel{\left\{x_{i}\right\}}{\longrightarrow} \quad\left(\operatorname{sig}_{i}:=G\right) ;
$$

for all $i \in\{0,1\}$ where $j=(i+1)$ mod 2. In other words, the algorithm strengthens the guard of $T C 1_{i}$ such that in the presence of faults, a signal does not turn green while the other one is also green.

In Step 4, consider the state predicate $Q_{n e w}-S_{1_{T C}}=\left(s i g_{0}=s i g_{1}=R\right) \wedge$ $\left(z_{0}, z_{1}>1\right)$. Similar to Step 3 , the algorithm adds recovery paths with the smallest possible time delay, which is the following action for either $i=0$ or $i=1$ :

$$
T C 6_{i}:: \quad\left(\operatorname{sig}_{i}=s i g_{j}=R\right) \wedge\left(z_{i}, z_{j}>1\right) \quad \stackrel{\left\{z_{i}\right\}}{\longrightarrow} \quad \text { skip; }
$$

It is straightforward to verify that by execution of $T C 6_{i}$, the program reaches the invariant $S_{T C}$ from where the program behaves correctly. Similar to Step 3 , the procedure Add_BoundedResponse may include the following additional actions:

$$
\begin{array}{lllll}
T C 7_{i}:: & \left(\operatorname{sig}_{i}=s i g_{j}=R\right) \wedge\left(z_{i}, z_{j}>1\right) \wedge\left(t_{2} \leq 7\right) & \stackrel{\left\{x_{i}\right\}}{\longrightarrow} & \left(\operatorname{sig}_{i}:=G\right) ; \\
T C 8_{i}:: & \left(\operatorname{sig}_{i}=s i g_{j}=R\right) \wedge\left(z_{i}, z_{j}>1\right) \wedge\left(t_{2} \leq 7\right) & \stackrel{\left\{y_{i}\right\}}{\longrightarrow} & \left(\operatorname{sig}_{i}:=Y\right) ; \\
T C 9_{i}:: & \left(\operatorname{sig}_{i}=s i g_{j}=R\right) \wedge\left(z_{i}, z_{j}>1\right) \wedge\left(t_{2} \leq 7\right) & \longrightarrow & \text { wait; }
\end{array}
$$

In the context of $T C$, in Step 5, the algorithm removes states from neither the fault-span nor the invariant, as $n s=\{\}$, and, hence, the algorithm finds the final solution in one iteration of the repeat-until loop.

\section{Related Work}

Our formulation of the synthesis problem is in spirit close to timed controller synthesis (e.g., [BDMP03,DM02,AM99,AMPS98]), where program and fault transitions may be modeled as controllable and uncontrollable actions, and game 
theory (e.g., [dAFH ${ }^{+}$03,FLM02]), where program and fault transitions may be modeled in terms of two players. In controller synthesis (respectively, game theory) the objective is to restrict the actions of a plant (respectively, an adversary) at each state through synthesizing a controller (respectively, a wining strategy) such that the behavior of the entire system always meets some safety and/or reachability conditions. Notice that the conditions $C 1 . . C 3$ in Problem Statement 1 precisely express this notion of restriction (also called language inclusion). Moreover, constraint $C 4$ implicitly implies that the synthesized program is not allowed to exhibit new finite computations, which is known as the nonblocking condition. Note, however, that there are several distinctions. First, in addition to safety and reachability constraints, our notion of fault-tolerance is also concerned with adding new bounded-time recovery behaviors to the given program as well, which is normally not a concern in controller synthesis and game theory. Secondly, unlike most game theoretic approaches, we do not consider turns between occurrence of program and fault transitions. Thirdly, in controller synthesis and game theory, a common assumption is that the existing program and/or the given specification must be deterministic which is not the case in our model.

Finally, we concentrate on safety properties typically used in specifying realtime systems (cf. Definition 8). As a result, the complexity of our synthesis techniques is often lower than the related work. For example, synthesis problems presented in $\left[\mathrm{dAFH}^{+}\right.$03,FLM02,AMPS98,AM99] are ExPTIME-complete and deciding the existence of a controller in [DM02,BDMP03] is 2ExpTIME-complete.

\section{$7 \quad$ Conclusion and Future Work}

In this paper, we focused on the problem of synthesizing fault-tolerant realtime programs that mask the occurrence of faults while providing boundedtime phased recovery. We modeled such phased recovery using bounded response properties of the form $(\neg S \mapsto \leq \theta) \wedge(Q \mapsto \leq \delta S)$ where $S$ is an invariant predicate and $Q$ is an intermediate recovery predicate. We showed that in general the problem is NP-complete in the size of locations of the input program. We also showed that if $S \subseteq Q$ and $Q$ is closed in execution of the output program then there exists a polynomial-time solution to the problem in the size of the input program's region graph.

Also, as discussed in Subsection 5.3, the designer can use the contrast between the complexity classes with slightly different problem specifications to determine if system requirements can be slightly modified for permitting automated synthesis. In particular, in Section 6, we argued that the alternate specification (where the problem is in $\mathrm{P}$ ) for the one-lane bridge problem considered in this paper may be acceptable to many designers. Also, as argued in that section, the modified specification can assist in partial automation of providing fault-tolerance with phased recovery. One of our future works in this area is to develop algorithms that utilize such a partial automation. 
We are currently working on other variations of the problem. One such variation is where $S \subseteq Q$, but $Q$ need not be closed in the output program. We conjecture that the complexity of this problem is exponential. We also plan to develop symbolic algorithms for synthesizing bounded-time phased recovery. In previous work, we have shown that such techniques are extremely effective in synthesizing distributed programs with state space of size $10^{30}$ and beyond [BK07].

\section{References}

[AD94] R. Alur and D. Dill. A theory of timed automata. Theoretical Computer Science, 126(2):183-235, 1994.

[AH97] R. Alur and T. A. Henzinger. Real-time system $=$ discrete system + clock variables. International Journal on Software Tools for Technology Transfer, 1(1-2):86-109, 1997.

[AM99] E. Asarin and O. Maler. As soon as possible: Time optimal control for timed automata. In Hybrid Systems: Computation and Control (HSCC), pages 19-30, 1999.

[AMPS98] E. Asarin, O. Maler, A. Pnueli, and J. Sifakis. Controller synthesis for timed automata. In IFAC Symposium on System Structure and Control, pages 469-474, 1998.

[AS85] B. Alpern and F. B. Schneider. Defining liveness. Information Processing Letters, 21:181-185, 1985.

[BDMP03] P. Bouyer, D. D'Souza, P. Madhusudan, and A. Petit. Timed control with partial observability. In Computer Aided Verification (CAV), pages 180192, 2003.

[BK06a] B. Bonakdarpour and S. S. Kulkarni. Automated incremental synthesis of timed automata. In International Workshop on Formal Methods for Industrial Critical Systems (FMICS), LNCS 4346, pages 261-276, 2006.

[BK06b] B. Bonakdarpour and S. S. Kulkarni. Incremental synthesis of fault-tolerant real-time programs. In International Symposium on Stabilization, Safety, and Security of Distributed Systems (SSS), LNCS 4280, pages 122-136, 2006.

[BK07] B. Bonakdarpour and S. S. Kulkarni. Exploiting symbolic techniques in automated synthesis of distributed programs with large state space. In IEEE International Conference on Distributed Computing Systems (ICDCS), pages $3-10,2007$.

$\left[\mathrm{dAFH}^{+}\right.$03] L. de Alfaro, M. Faella, T. A. Henzinger, R. Majumdar, and M. Stoelinga. The element of surprise in timed games. In International Conference on Concurrency Theory (CONCUR), 2003.

[DM02] D. D'Souza and P. Madhusudan. Timed control synthesis for external specifications. In Symposium on Theoretical Aspects of Computer Science (STACS), pages 571-582, 2002.

[FLM02] M. Faella, S. LaTorre, and A. Murano. Dense real-time games. In Logic in Computer Science (LICS), pages 167-176, 2002.

[Hen92] T. A. Henzinger. Sooner is safer than later. Information Processing Letters, 43(3):135-141, 1992. 